\title{
Predictive value of coagulation profiles for both initial and repeated immunoglobulin resistance in Kawasaki disease: $A$ prospective cohort study
}

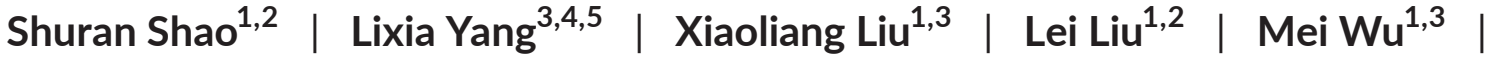 \\ Yuxin Deng ${ }^{6}$ | Hongyu Duan ${ }^{1,3,4,5}$ | Yifei Li ${ }^{1,3,4,5}$ | Yimin Hua ${ }^{1,3,4,5}$ | Lili Luo \\ Kaiyu Zhou ${ }^{1,3,4,5}$ | Chuan Wang ${ }^{1,3,4,5}$

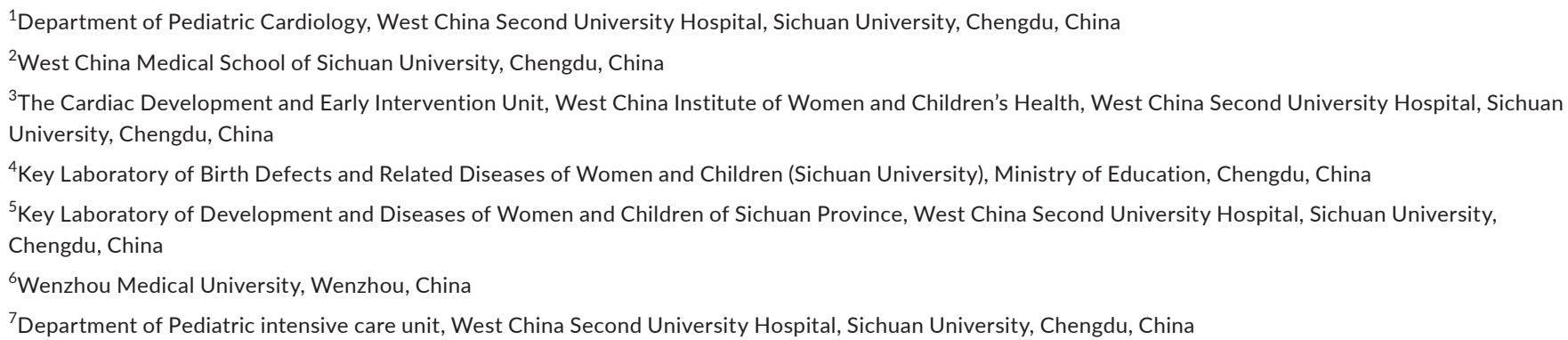

\section{Correspondence}

Kaiyu Zhou, Department of Pediatric Cardiology, West China Second University Hospital, Sichuan University, No. 20, Section 3, South Renmin Road, Chengdu, Sichuan, 610041, China.

Email: kaiyuzhou313@163.com

Chuan Wang, Department of Pediatric Cardiology, West China Second University Hospital, Sichuan University, No. 20, 3rd section, South Renmin Road Chengdu, 610041, China.

Email: 805101396@qq.com

\section{Funding information}

This work was supported by the National Key R\&D Program of China (No. 2018YFC1002301), National Natural Science Foundation of China (No. 81800288 , No. 82070324 , No. 81971457 , and No. 81701499), Science-Technology Support Plan Projects in Sichuan Province

\begin{abstract}
Background: Intravenous immunoglobulin (IVIG) resistance prediction remains substantial in Kawasaki disease (KD), with limited data on the predictive value of coagulation profile for IVIG resistance, particularly for repeated IVIG resistance. Therefore, the aim of our study was to testify the predictive validity of coagulation profile for both initial IVIG resistance and repeated IVIG resistance in KD.

Methods: A total of $385 \mathrm{KD}$ patients were prospectively recruited between April 2015 and May 2019. Coagulation and other profiles were evaluated between the IVIG-responsive and IVIG-resistant groups. Multivariate logistic regression analysis was applied to determine the association between coagulation profiles and IVIG resistance. ROC curves analysis was further performed to assess the validity of coagulation profiles in predicting both initial IVIG resistance and repeated IVIG resistance. Results: Prothrombin time (PT), activated partial thromboplastin time (APTT), international normalized ratio (INR), fibrinogen degradation products (FDPs), and D-dimer were significantly increased in the initial IVIG-resistant group with antithrombin III
\end{abstract}

Abbreviations: ALT, serum alanine aminotransferase; APTT, activated partial thromboplastin time; AST, aspartate aminotransferase; ATIII, antithrombin III; AUC, area under the curve; CAL, coronary artery lesion; $\mathrm{Cl}$, confidence interval; CRP, C-reactive protein; FDP, fibrinogen degradation products; INR, international normalized ratio; IVIG, intravenous immunoglobulin; KD, Kawasaki disease; NLR, neutrophil-to-lymphocyte ratio; OR, odds ratio; PT, prothrombin time; ROC, receiver operating characteristic; TB, total bilirubin; TT, thrombin time.

Shao and Yang contributed equally to this study.

This is an open access article under the terms of the Creative Commons Attribution-NonCommercial License, which permits use, distribution and reproduction in any medium, provided the original work is properly cited and is not used for commercial purposes.

(c) 2021 The Authors. Pediatric Allergy and Immunology published by European Academy of Allergy and Clinical Immunology and John Wiley \& Sons Ltd. 
(No. 2017SZ0117, No. 2019YFS0243, No. 2020YFS0101, and No. 2020YJ0234).

Editor: Marina Atanaskovic-Markovic
(ATIII) and thrombin time (TT) significantly reduced. Meanwhile, ATIII was declined markedly in repeated IVIG-resistant patients. Multivariate logistic regression analysis showed that PT, APTT, D-dimer, and ATIII were independent risk factors for predicting initial IVIG resistance and ATIII for predicting repeated IVIG-resistant patients with KD. PT, APTT, D-dimer, and ATIII cutoff values of 13.95 s, 41.15 s, 1.48 mg/L, and $89.5 \%$ yielded sensitivities of $73 \%, 32 \%, 71 \%$, and $81 \%$, and specificities of $55 \%$, $88 \%, 62 \%$, and 51\% for predicting initial IVIG resistance, respectively. The cutoff value of ATIII for predicting repeated IVIG resistance was $68.5 \%$, with sensitivity of $71 \%$ and specificity of $55 \%$.

Conclusions: KD patients who have hypercoagulation during the acute phase might be at higher risk of developing IVIG resistance.

\section{KEYWORDS}

Antithrombin III, coagulation profile, immunoglobulin resistance, kawasaki disease, prediction

\section{Key Message}

Currently, studies elucidating the association between the coagulation profile and Kawasaki disease (KD), especially with regard to IVIG resistance, are limited. To the best of our knowledge, this is the first study to clarify the association between coagulation and Intravenous immunoglobulin (IVIG) resistance in patients with KD based on a relatively large clinical dataset. Our results suggest that patients with impaired coagulation who are resistant to IVIG (initial and/or repeated) may need more aggressive treatment to reduce the likelihood of developing coronary artery lesions (CALs).

\section{$1 \mid$ INTRODUCTION}

Kawasaki disease (KD) is an acute vasculitis that is the leading cause of acquired heart disease in children, with approximately $15 \%-20 \%$ of patients with KD suffering intravenous immunoglobulin (IVIG) resistance. ${ }^{1}$ For children who do not respond to initial IVIG treatment, repeated IVIG infusion is recommended by many experts. ${ }^{2}$ However, approximately $10 \%$ of patients are resistant to both initial IVIG therapy and repeated IVIG therapy, ${ }^{3}$ and thus have a higher risk of coronary artery lesions (CALs). Therefore, early identification of both initial IVIG resistance and repeated IVIG resistance is of great importance to reduce CALs, and, most importantly, lower medical costs.

It has long been known that inflammation triggered by acute infection can lead to the activation of the coagulation system by upregulating the expression of cytokines. ${ }^{4}$ In an investigation of critically ill patients, Ogura et $\mathrm{al}^{5}$ found that abnormal coagulation was associated with increased systemic inflammatory response syndrome scores. In addition, the relationship between the activation of the immune system and coagulation system is evident in systemic autoimmune or immune-mediated diseases. ${ }^{6}$ Therefore, the balance between coagulation and the fibrinolytic system may be disturbed during the acute stages of KD, particularly in patients with IVIG resistance, since KD vasculitis is accompanied by an increase in inflammatory cells and cytokines, and IVIG resistance may reflect a more severe inflammatory immune condition. Indeed, several studies have previously explored the coagulation profiles in patients with KD. ${ }^{7-12}$ However, most of them focused on the changes in coagulation profiles before and after IVIG in $\mathrm{KD},{ }^{10}$ the role of coagulation profiles in distinguishing atypical KD from bacterial cervical lymphadenitis, ${ }^{12}$ and in predicting the occurrence of CALs or cardiac complications ${ }^{9-11}$. The role of coagulation profile in initial IVIG resistance prediction was only explored in two studies. ${ }^{7,8}$ Nevertheless, both of them were limited by small sample size and retrospective nature. Most importantly, the results from them were inconsistent and the predictive value of ATIII has never been explored before. Furthermore, data on the validity of coagulation profiles in repeated IVIG response prediction are lacking. Therefore, a large prospective cohort study was conducted to assess the predictive validity of the serum coagulation profile, including prothrombin time (PT), activated partial thromboplastin time (APTT), thrombin time (TT), international normalized ratio (INR), and fibrinogen, D-dimer, fibrin degradation products (FDPs), and antithrombin III (ATIII) levels for identifying patients with KD at risk of both initial IVIG resistance and repeated IVIG resistance.

\section{2 | MATERIALS AND METHODS}

\section{1 | Subjects}

Patients with KD were prospectively recruited between April 2015 and May 2019 at our hospital. A diagnosis of KD relied on 
the standards recommended by the American Heart Association's Scientific Statement for the diagnosis, treatment, and long-term management of $K \mathrm{D}^{13}$ and was confirmed by two experienced pediatricians (at least one was a KD specialist). Informed written consent was obtained from the parents after the nature of the study had been fully explained to them. The University Ethics Committee on Human Subjects at Sichuan University approved the study.

Exclusion criteria included known patients with congenital or chronic hematologic disease affecting the coagulation cascade; patients with end-stage renal disease requiring dialysis, acute or chronic liver failure, and autoimmune disease; patients who had undergone surgery recently; patients with infectious or inflammatory diseases; and patients who received oral anticoagulant or heparin therapy. A total of 520 patients diagnosed with KD were initially screened for participation in this study. Of these, patients who had received initial IVIG treatment at other medical facilities $(n=87)$, received IVIG treatment within 10 days of fever onset $(n=12)$, or cases where IVIG treatment was initiated before blood sampling ( $n=17$ ) were excluded. Another 19 patients were excluded because of incomplete laboratory data or lack of follow-up results (Figure 1). Finally, data from 385 patients were analyzed.

All patients received $2 \mathrm{~g} / \mathrm{kg}$ of IVIG for 24 hours and $30-50 \mathrm{mg} /$ $\mathrm{kg} / \mathrm{day}$ of aspirin until they were afebrile. Initial IVIG resistance was defined as recurrent or persistent fever or other clinical signs

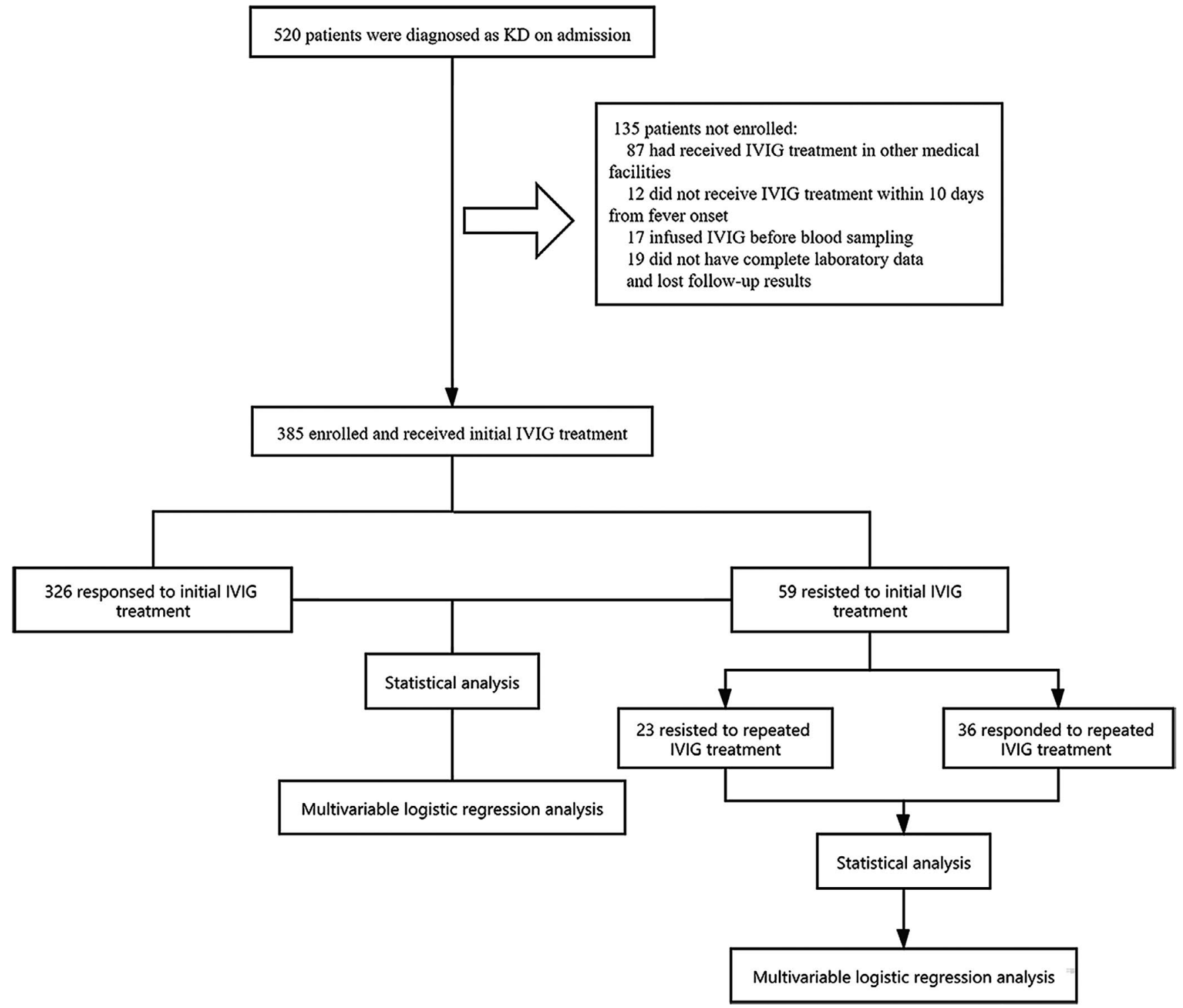

FIGURE 1 The flowchart of our prospective study. A total of 520 patients diagnosed with KD were initially screened for participation in this study. Of these, patients who had received initial IVIG treatment at other medical facilities $(\mathrm{n}=87$ ), received IVIG treatment within 10 days of fever onset $(n=12)$, or cases where IVIG treatment was initiated before blood sampling $(n=17)$ were excluded. Another 19 patients were excluded because of incomplete laboratory data or lack of follow-up results. Finally, data from 385 patients were analyzed. Of the 385 patients, 326 (84.7\%) responded to initial IVIG treatment, whereas 59 (15.3\%) did not respond. Of the 59 patients with initial IVIG resistance, 23 did not respond to repeated IVIG treatment and received pulse intravenous methylprednisolone infusion. Then, data analysis and multivariate logistic regression analysis were performed in these groups 
of KD for at least 36 hours but not longer than 7 days after initial IVIG treatment. For patients with initial IVIG resistance, the second IVIG dose (2 $\mathrm{g} / \mathrm{kg}$ given as a single intravenous infusion) was administered according to expert consensus on the diagnosis and treatment of $K D$ in China. Furthermore, pulse intravenous methylprednisolone (10-30 mg/kg/day for three consecutive days) followed by oral prednisone ( $2 \mathrm{mg} / \mathrm{kg} /$ day) tapered over seven days was additionally administered if the patient had recurrent or persistent fever even after the second IVIG administration. No patients received any additional treatment such as infliximab, plasma exchange, or cytotoxic agents.

According to institutional protocol, standardized echocardiograms were obtained from patients by the same experienced pediatric ultrasonologists during the acute/subacute phase and 6-8 weeks later in the cardiology clinic during follow-up evaluations until the coronary artery abnormalities were resolved. CALs were defined based on the normalization of dimensions for body surface area as Z-scores (standard deviation units from the mean, normalized for body surface area): no involvement (Z-score $<2.0$ ), dilation (Z-score $\geq 2.0$ to $<2.5$ ), and aneurysm (Z-score $\geq 2.5$; Z-score $\geq 10$ for giant aneurysms) of the coronary arteries depending on the maximal internal diameters of the right coronary, left anterior descending, and left circumflex coronary arteries.

Patients were subsequently categorized into two groups depending on whether they responded to the initial IVIG treatment (initial IVIG-responsive group, $\mathrm{n}=326$; initial IVIG-resistant group, $\mathrm{n}=59$ ). The initial IVIG-resistant group was further divided into two subgroups based on the effectiveness of repeated IVIG treatment [repeated IVIG-responsive group $(n=36)$ and repeated IVIGresistant group $(\mathrm{n}=23)]$.

\section{2 | Laboratory measurements}

Coagulation analyses of the patient's plasma, including PT, APTT, TT, fibrinogen, D-dimer, FDP, international normalized ratio, and ATIII activity, were performed before initial IVIG within 10 days from fever onset. Standard coagulation measurements were performed with a CoagXL-automated coagulometer (Diagon Ltd.) using reagents from Diagon.

\subsection{Statistics}

Data analyses were performed using SPSS version 21.0 (SPSS Inc.) Quantitative data are presented as the medians with the 25th and 75th percentiles in square brackets, while qualitative data are expressed as the number or percentage as appropriate. A chi-squared test and unpaired Student's $t$ test or Mann-Whitney $U$ test were used to compare the demographic characteristics, clinical manifestations, and laboratory data. Significant indicators from univariate analysis were then subjected to multivariate logistic regression analysis to identify independent predictors of IVIG resistance. Receiver operating characteristic (ROC) curve analysis was used to evaluate the value of coagulative biomarkers for predicting the development of initial and repeated IVIG resistance. A $P$ value $<.05$ was considered statistically significant.

\section{3 | RESULTS}

\section{1 | Subjects}

CALs were observed in 47 patients (12.2\%), while transient pericardial effusion, valve regurgitation, cardiac enlargement, and ventricular systolic dysfunction were noted in $11,40,38$, and 2 children, respectively. No significant difference was found in serum coagulation profiles between the CAL and non-CAL groups. (Supplemental material 1).

\subsection{Serum coagulation levels for predicting initial IVIG resistance}

As shown in Table 1, there were no significant differences in age, sex, the day of illness before IVIG treatment, sampling day of illness, and typical clinical manifestations between the initial IVIG-responsive and IVIG-resistant groups. As for other cardiac complications, the percentage of patients with cardiac enlargement and pericardial effusion was found to be significantly higher in the initial IVIG-resistant group ( $P=.003$ and $P=.016$, respectively). Initial IVIG-resistant patients had much higher incidence of CALs, with substantial higher level of serum C-reactive protein (CRP), neutrophil-to-lymphocyte ratio (NLR), serum total bilirubin (TB), serum alanine aminotransferase (ALT), creatinine, and urea nitrogen, but with lower hemoglobin, platelet count, albumin, sodium, and potassium before the initial IVIG treatment (all $P<.05$ ). No significant differences were found in the white blood cell count, erythrocyte sedimentation rate, and aspartate aminotransferase (AST) between the two groups.

The effect of KD on the coagulation system was evaluated by assessing the coagulation profiles of initial IVIG-responsive and IVIG-resistant subjects. The PT (14.3 [13.6-15.5] s vs 13.8 [1314.4] s, $P=.005)$ and APTT (35.3 [31.9-44.7] s vs 34.2 [30.7-38.1] $\mathrm{s}, P=.006)$ were significantly longer, and TT (16.0[15.8-16.4]s vs $16.5[16-16.9] \mathrm{s}, P<.001)$ was significantly shorter in the initial IVIGresistant group, with significantly higher INR (1.19[1.13-1.30] vs 1.13[1.06-1.20], $P<.001)$, FDP (8.7[6.2-12.5]ug/mL vs 5.9[3.8-9.8] ug $/ \mathrm{mL}, P<.001)$, D-dimer levels $(2.1$ [1.20-2.82] mg/L vs 1.2 [0.8$2.07] \mathrm{mg} / \mathrm{L}, P=.020)$, and ATIII activity (81\% [65\%-88\%] vs $89 \%$ [81\%-100\%], $P<.001$ ) (Table 1).

\subsection{Multivariate logistic regression and ROC curve analysis for predicting initial IVIG resistance}

To not affect the prediction efficiency, the correlation between univariate factors and coagulation profiles was tested by Pearson's 
TABLE 1 Comparison of clinical data between the initial intravenous immunoglobulin (IVIG)-resistant and IVIG-responsive groups in Kawasaki disease

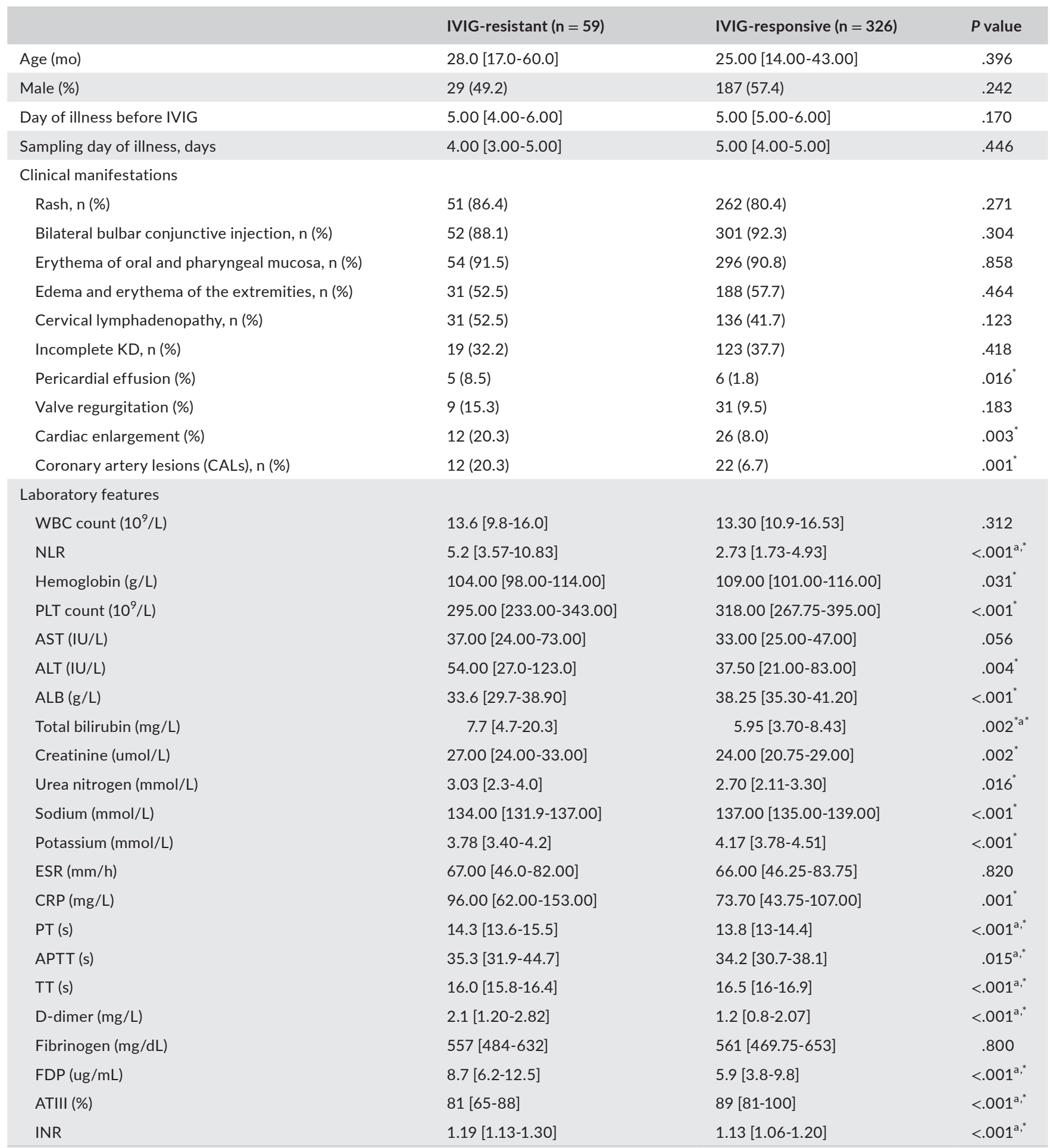

The data are presented as the median with the 25 th and 75 th percentiles in square brackets for continuous variables and as the percentage for the categorical variables.

Abbreviations: ALB, albumin; ALT, alanine aminotransferase; APTT, activated partial thromboplastin time; AST, aspartate aminotransferase; AT, antithrombin; CALs, coronary artery lesionsCRP, C-reactive protein; ESR, erythrocyte sedimentation rate; FDP, fibrin degradation products; INR, international normalized ratio; NLR, neutrophil-to-lymphocyte ratio; PLT, platelet; PT, prothrombin time; TT, total thrombin time; WBC, white blood cell.

${ }^{a}$ Variables between the two groups were compared by the Mann-Whitney $\mathrm{U}$ test due to abnormal data distribution.

${ }^{*}$ Statistically significant $(P<.05)$ 
TAB LE 2 A multivariate logistic regression model for initial and repeated intravenous immunoglobulin (IVIG) resistance in Kawasaki disease

\begin{tabular}{|c|c|c|c|c|c|c|}
\hline Variates & $\boldsymbol{\beta}$ & SE & Walds & $P$ value & OR & $95 \% \mathrm{Cl}$ \\
\hline Platelet & 0.005 & 0.002 & 7.523 & $.006^{*}$ & 1.01 & $1.00-1.01$ \\
\hline Urea nitrogen & -0.168 & 0.125 & 1.804 & .179 & 0.85 & $0.66-1.08$ \\
\hline ALT & -0.002 & 0.001 & 2.365 & .124 & 0.99 & $0.99-1.00$ \\
\hline \multicolumn{7}{|c|}{ Initial IVIG resistance-APTT } \\
\hline Platelet & 0.004 & 0.002 & 1.323 & $.038^{*}$ & 1.00 & $1.00-1.01$ \\
\hline Creatinine & -0.019 & 0.022 & 0.707 & .400 & 0.98 & $0.94-1.03$ \\
\hline Urea nitrogen & -0.190 & 0.133 & 2.017 & .152 & 0.83 & $0.64-1.07$ \\
\hline \multicolumn{7}{|c|}{ Initial IVIG resistance-D-dimer } \\
\hline Platelet & 0.004 & 0.002 & 4.257 & $.039^{*}$ & 1.00 & $1.00-1.01$ \\
\hline Creatinine & -0.016 & 0.023 & 0.532 & .466 & 0.98 & $0.94-1.03$ \\
\hline Urea nitrogen & -0.175 & 0.133 & 1.746 & .186 & 0.84 & $0.65-1.09$ \\
\hline ALT & 0.000 & 0.001 & 0.350 & .554 & 1.00 & $0.99-1.00$ \\
\hline Potassium & 0.953 & 0.300 & 10.06 & $.002^{*}$ & 2.59 & $1.44-4.67$ \\
\hline Total bilirubin & -0.021 & 0.012 & 3.419 & .064 & 0.98 & $0.96-1.00$ \\
\hline D-dimer & -0.129 & 0.063 & 4.237 & $.040^{*}$ & 0.88 & $0.78-0.99$ \\
\hline \multicolumn{7}{|c|}{ Initial IVIG resistance-ATIII } \\
\hline Platelet & 0.004 & 0.002 & 3.751 & .053 & 1.00 & $1.00-1.01$ \\
\hline Creatinine & -0.027 & 0.023 & 1.396 & .237 & 0.97 & $0.93-1.02$ \\
\hline Urea nitrogen & -0.173 & 0.134 & 1.667 & .197 & 0.84 & $0.65-1.09$ \\
\hline ALT & -0.001 & 0.001 & 0.303 & .582 & 1.00 & $1.00-1.01$ \\
\hline Potassium & 0.782 & 0.306 & 6.512 & $.011^{*}$ & 2.19 & $1.12-3.98$ \\
\hline Total bilirubin & -0.021 & 0.012 & 2.915 & .088 & 0.98 & $0.96-1.00$ \\
\hline TT & 0.287 & 0.226 & 1.618 & .203 & 1.33 & $0.86-2.07$ \\
\hline \multicolumn{7}{|c|}{ Initial IVIG resistance-INR } \\
\hline NLR & -0.476 & 0.491 & 0.941 & .332 & 0.62 & $0.24-1.63$ \\
\hline C-creative protein & 0.000 & 0.004 & 0.000 & .99 & 1.00 & $0.99-1.01$ \\
\hline Platelet & 0.004 & 0.002 & 4.000 & $.046^{*}$ & 1.00 & $1.00-1.01$ \\
\hline Creatinine & -0.012 & 0.023 & 0.287 & .592 & 0.99 & $0.92-1.03$ \\
\hline Urea nitrogen & -0.070 & 0.133 & 0.274 & .600 & 0.93 & $0.72-1.21$ \\
\hline ALT & -0.001 & 0.001 & 0.160 & .689 & 1.00 & $1.00-1.01$ \\
\hline Potassium & 0.706 & 0.325 & 4.712 & $.030^{*}$ & 2.03 & $1.07-3.83$ \\
\hline Sodium & 0.086 & 0.048 & 3.222 & .073 & 1.09 & $0.99-1.20$ \\
\hline
\end{tabular}


TABLE 2 (Continued)

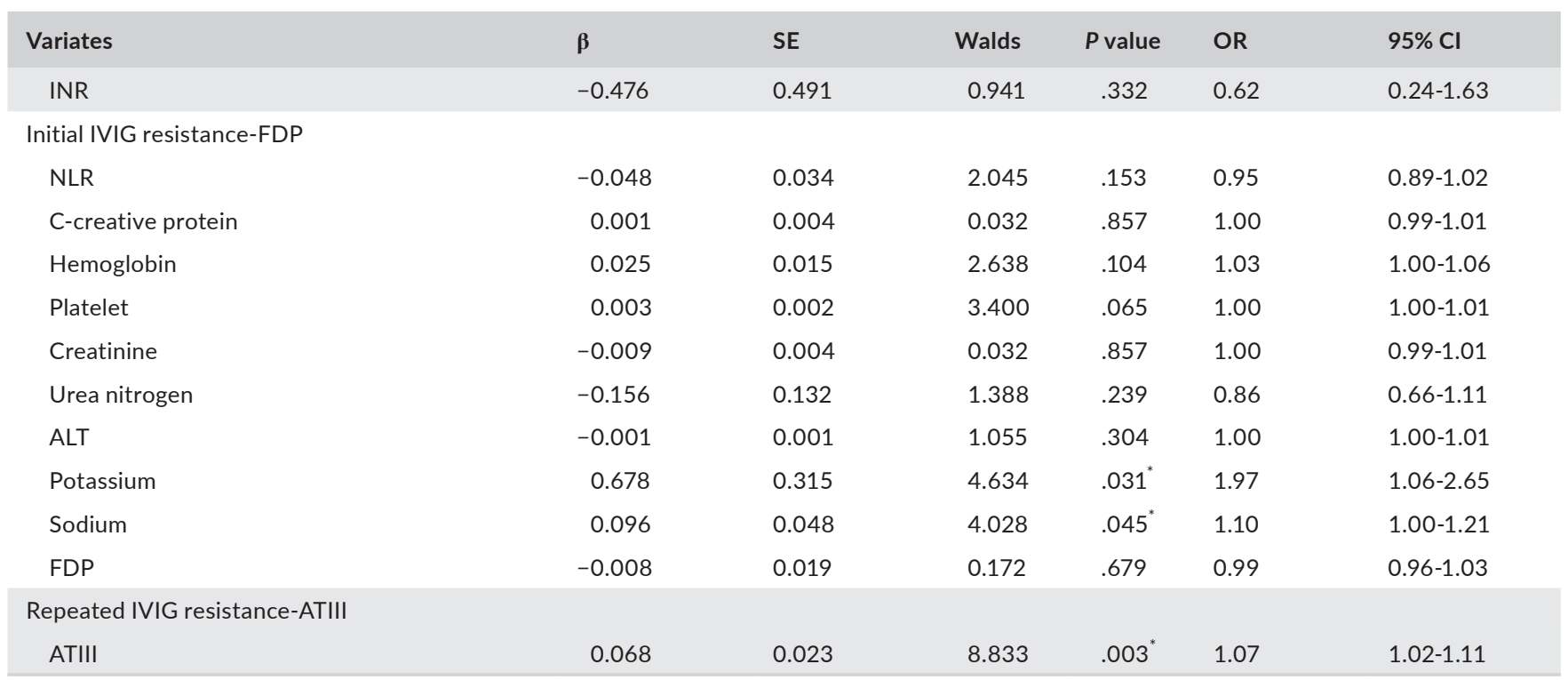

Abbreviations: ALT, alanine aminotransferaseAPTT, activated partial thromboplastin time; ATIII, antithrombin III; FDP, fibrin degradation products; INR, international normalized ratio; IVIG, intravenous immunoglobulin; PT, prothrombin time; TT, total thrombin time.

*Statistically significant $(P<.05)$

analysis. The strong variables were removed from the model (Supplemental material 2). Multivariate logistic regression analysis showed that longer PT, APTT, higher D-dimer, and lower ATIII activity were independent risk factors for initial IVIG resistance in patients with KD, whereas INR, TT, and FDP failed to reach significant difference in the multivariate logistic regression analysis (Table 2).

The validity of the serum coagulation profile in predicting initial IVIG resistance in patients with KD was assessed using ROC curve analysis. The areas under the curve (AUC) of the various variables for predicting IVIG resistance were 0.662, 0.599, 0.684, and 0.706 for PT, APTT, D-dimer, and ATIII, respectively. The PT, APTT, D-dimer, and ATIII cutoff values of $13.95 \mathrm{~s}, 41.15 \mathrm{~s}, 1.48 \mathrm{mg} / \mathrm{L}$, and $89.5 \%$ yielded sensitivities of $73 \%, 32 \%, 71 \%$, and $81 \%$, and specificities of $55 \%, 88 \%, 62 \%$, and $51 \%$ for predicting initial IVIG resistance, respectively (Table 3 and Figure $2 \mathrm{~A}$ ).

\subsection{Serum coagulation levels for predicting repeated IVIG resistance}

A comparison of clinical data between the repeated IVIG-responsive ( $n=33$ ) and repeated IVIG-resistant $(n=26)$ groups is shown in Table 4. The repeated IVIG-resistant subjects had lower ATIII activity than those of repeated IVIG-responsive subjects (65\% [60\%-81\%] vs $84 \%$ [76.23\%-89.75\%], $P=.001$ ). There was no significant difference in any other examined parameters between the two groups. Multivariate logistic regression analysis showed that lower ATIII activity was independent risk factor for repeated IVIG resistance in patients with KD (Table 2).

\section{5 | ROC curve analysis for predicting repeated IVIG resistance}

ROC curves were used to calculate the $A U C$ and predictive values of each coagulation parameter (Table 3 and Figure 2B). The AUC of ATIII for predicting repeated-KD patients was 0.753 . In repeated-KD patients, the cutoff value of ATIII was $68.5 \%$, which yielded a sensitivity of $71 \%$ and specificity of $55 \%$ for predicting repeated IVIG resistance.

\section{DISCUSSION}

To the best of our knowledge, this is the first study to clarify the association between coagulation and IVIG resistance in patients with KD based on a relatively large clinical dataset. In this prospective study, we support the hypothesis that there are marked differences in coagulation profiles, including longer PT and APTT, higher INR, FDP, D-dimer levels, shorter TT, and lower ATIII activity, that may predict initial IVIG resistance. In addition, using multivariate logistic regression analysis, we found that longer PT, APTT, higher D-dimer, and lower ATIII activity before initial IVIG were significant independent risk factors for initial IVIG resistance. Furthermore, significantly lower ATIII activity was found in patients with repeated IVIG resistance, and multivariate logistic regression analysis showed that lower ATIII activity was independent risk factor for predicting repeated IVIG resistance. Our results suggest that patients with impaired coagulation who are resistant to IVIG (initial and/or repeated) may need more aggressive treatment to reduce the likelihood of developing CALs. 
TABLE 3 The validity of coagulation profiles in predicting initial and repeated intravenous immunoglobulin (IVIG) resistance

\begin{tabular}{|c|c|c|c|c|c|c|c|c|c|c|c|}
\hline $\begin{array}{l}\text { Initial IVIG } \\
\text { resistance }\end{array}$ & Diagnostic test & Gold stan & ard & & Sen & Spe & PPV & NPV & $\begin{array}{l}\text { Diagnostic } \\
\text { accuracy }\end{array}$ & OR $(95 \% \mathrm{CI})$ & $P$ \\
\hline \multirow{6}{*}{$\begin{array}{l}\text { Total group } \\
(\mathrm{n}=385)\end{array}$} & $\mathrm{PT} \geq 13.95 \mathrm{~s}$ & Positive & 43 & 147 & 0.73 & 0.55 & 0.23 & 0.92 & 0.58 & $3.27(1.77-6.05)$ & $<.001^{*}$ \\
\hline & \multirow[t]{2}{*}{ APTT $\geq 41.15 \mathrm{~s}$} & Positive & 19 & 40 & \multirow[t]{2}{*}{0.32} & \multirow[t]{2}{*}{0.88} & \multirow[t]{2}{*}{0.32} & \multirow[t]{2}{*}{0.88} & \multirow[t]{2}{*}{0.79} & \multirow[t]{2}{*}{$3.40(1.79-6.43)$} & \multirow[t]{2}{*}{$<.001^{*}$} \\
\hline & & Negative & 40 & 286 & & & & & & & \\
\hline & $\begin{array}{l}\text { D-dimer } \\
\geq 1.48 \mathrm{mg} / \mathrm{I}\end{array}$ & Negative & 17 & 201 & 0.71 & 0.62 & 0.25 & 0.92 & 0.63 & $3.97(2.17-7.28)$ & $<.001^{*}$ \\
\hline & \multirow[t]{2}{*}{ ATIII $\leq 89.5 \%$} & Positive & 48 & 160 & \multirow[t]{2}{*}{0.81} & \multirow[t]{2}{*}{0.51} & \multirow[t]{2}{*}{0.23} & \multirow[t]{2}{*}{0.94} & \multirow[t]{2}{*}{0.56} & \multirow[t]{2}{*}{$4.53(2.27-9.03)$} & \multirow[t]{2}{*}{$<.001^{*}$} \\
\hline & & Negative & 11 & 166 & & & & & & & \\
\hline \multirow{2}{*}{$\begin{array}{l}\text { Repeated group } \\
(n=59)\end{array}$} & \multirow[t]{2}{*}{ ATIII $\leq 68.5 \%$} & Positive & 42 & 146 & \multirow[t]{2}{*}{0.71} & \multirow[t]{2}{*}{0.55} & \multirow[t]{2}{*}{0.22} & 0.91 & 0.58 & $3.05(1.67-5.57)$ & $<.001^{*}$ \\
\hline & & Negative & 17 & 180 & & & & & & & \\
\hline
\end{tabular}

Abbreviations: APTT, activated partial thromboplastin time; AT, antithrombin; IVIG, intravenous immunoglobulin;PT, prothrombin time. $\mathrm{Cl}$, confidence ratio; NPV: negative predictive value; OR, odds ratio; PPV: positive predictive value; Sen: sensitivity; Spe: specificity

*Statistically significant $(P<.05)$.

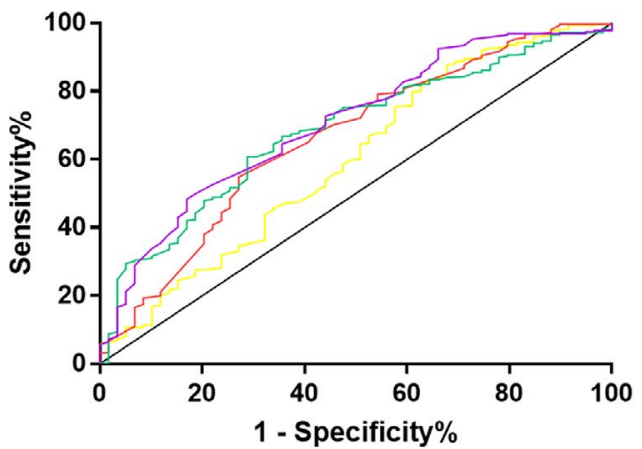

ROC curve for predicting initial IVIG resistacne

(A)
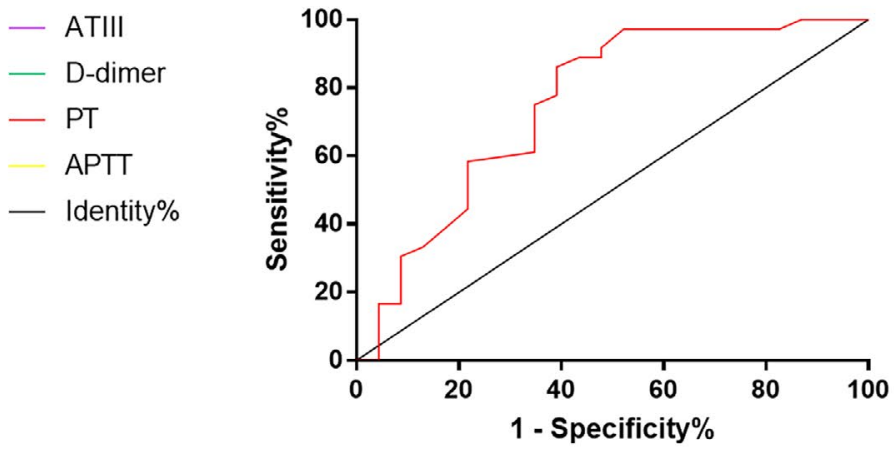

ROC curve for predicting repeated IVIG resistance

(B)

FIGURE 2 The receiver operating characteristic (ROC) curve for coagulation profiles in predicting initial and repeated IVIG resistance. (A) ROC curve for coagulation profiles [prothrombin time (PT), activated partial thromboplastin time (APTT), antithrombin III (ATIII), Ddimer] in predicting initial IVIG resistance. (B) ROC curve for ATIII in predicting repeated IVIG resistance

Although the underlying mechanisms regarding the impaired blood coagulation in KD are not entirely clear, several explanations could be hypothesized. Firstly, the pathophysiologic basis of KD is systemic, medium-sized vasculitis with inflammatory hypercytokinemia. ${ }^{14}$ The inflammatory cytokines such as TNF- $\alpha$ and IL- 6 would excessively release in the acute phase of $\mathrm{KD} .{ }^{15,16}$ The cytokine profile may reflect the disease severity and is associated with the development of IVIG resistance. ${ }^{17}$ In the present study, higher NLR, AST, ALT, and CRP indicate more severe inflammation of patients in the IVIG-resistant group than those in the IVIG-responsive group. Moreover, vascular inflammation enhances vascular permeability. Higher prevalence of pericardial effusion of the IVIG-resistant group might reflect more severe TNF- $\alpha$-mediated vasculitis. ${ }^{15}$ The clinical importance of the interaction between inflammation and coagulation is widely accepted recently. ${ }^{18}$ Higher pro-inflammatory cytokine levels including TNF- $\alpha$, IL-6, and IL-8 were found in those with higher D-dimer levels. TNF- $\alpha$, one of the leading cytokines of KD, could induce vascular endothelial cell injury via oxidative stress, inflammation, and apoptosis. ${ }^{19}$ Vascular endothelium is luminary covered by network of membrane-bound proteoglycans and glycoproteins (glycocalyx). ${ }^{20,21}$ If glycocalyx is damaged by KD vasculitis, anticoagulant effect of vascular endothelium is impaired followed by abnormal platelet aggregation, hypercoagulable state, and hyperfibrinolysis. ${ }^{22}$ Therefore, the impaired blood coagulation could reflect the severity of vascular damage in KD and thereby could be used as useful markers for IVIG resistance prediction.

Secondly, prolonged PT and APTT could result from decreased synthesis and/or increased consumption of coagulation factors. ${ }^{23}$ Therefore, the impaired production of coagulation factors due to liver damage in KD might also be involved in abnormal coagulable state since most of the coagulation factors are synthesized in the liver. Indeed, elevated serum liver enzymes, hypoalbuminemia, and hyperbilirubinemia were more severe in the initial IVIG-resistant group in comparison with the initial IVIG-responsive group in our 
TAB LE 4 Comparison of clinical data between the repeated intravenous immunoglobulin (IVIG)-resistant and IVIG-responsive groups in Kawasaki disease

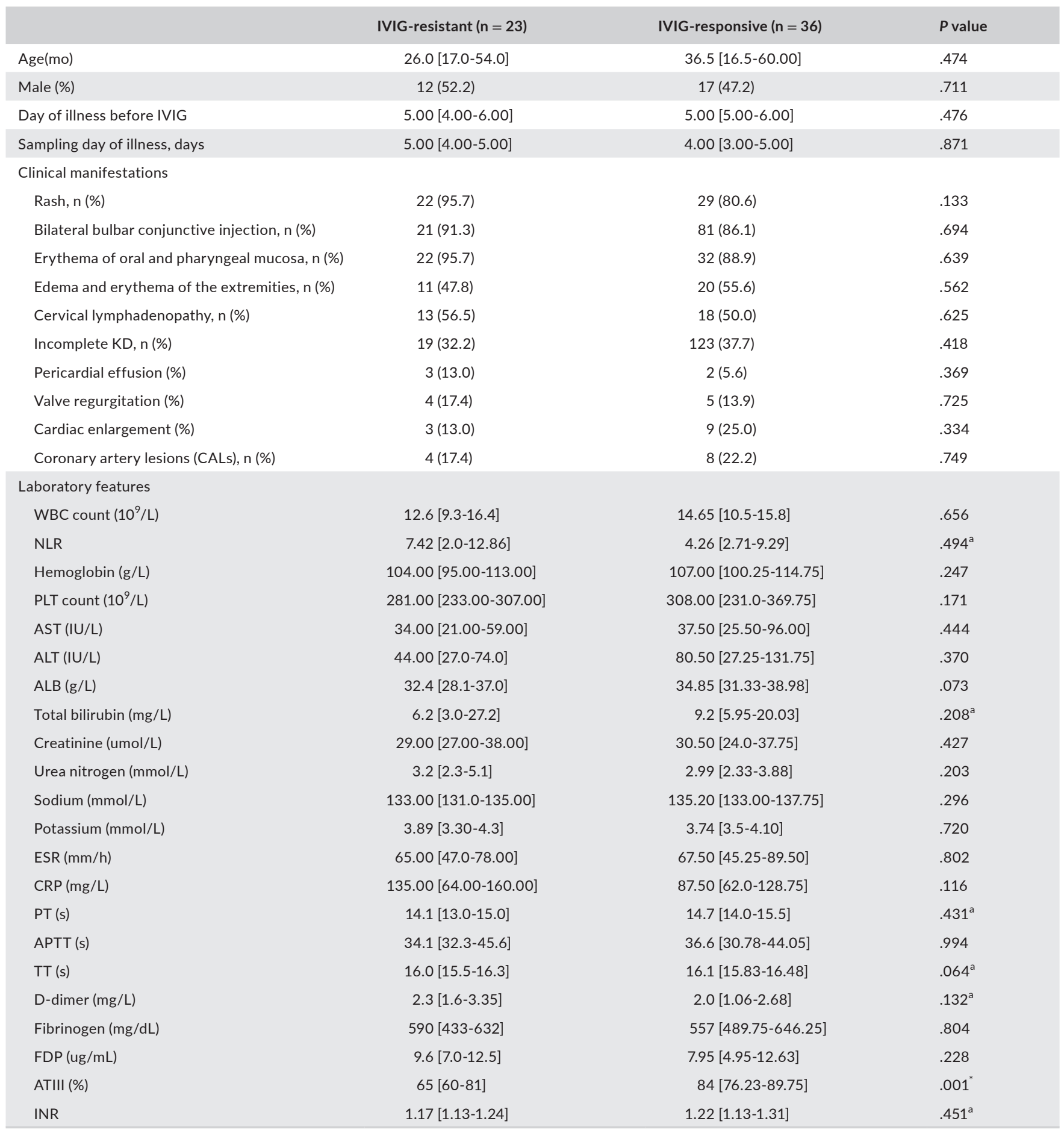

The data are presented as the median with the 25 th and 75 th percentiles in square brackets for continuous variables and as the percentage for the categorical variables.

Abbreviations: ALB, albumin; ALT, alanine aminotransferase; APTT, activated partial thromboplastin time; AST, aspartate aminotransferase; AT, antithrombin; CALs, coronary artery lesions; CRP, C-reactive protein; ESR, erythrocyte sedimentation rate; FDPs, fibrin degradation products; INR, international normalized ratio; NLR, neutrophil-to-lymphocyte ratio; PLT, platelet; PT, prothrombin time; TT, total thrombin time; WBC, white blood cell.

${ }^{a}$ Variables between the two groups were compared by the Mann-Whitney $U$ test due to abnormal data distribution.

*Statistically significant $(P<.05)$. 
study. Additionally, a previous study conducted by Wei-Xing Kong ${ }^{8}$ in 2019 has found the serum level of cholinesterase, which could reflect the hepatic synthetic capacity, was significantly decreased in IVIG non-responders. Collectively, the prolonged PT and APTT in the initial IVIG-resistant group might also, to some extent, be attributable to the more severe hepatic dysfunction in KD.

Several studies have previously explored the coagulation profiles in patients with KD. ${ }^{7-12,24,25}$ However, most of them focused on the changes in coagulation profiles before and after IVIG in KD, ${ }^{10}$ the role of coagulation profiles in distinguishing atypical KD from bacterial cervical lymphadenitis, ${ }^{12}$ and in predicting the occurrence of CALs or cardiac complications. ${ }^{9-11}$. Chen et $\mathrm{al}^{24}$ in 2015 noticed that the levels of fibrinogen and D-dimer were significantly elevated in KD patients compared with healthy children. Yoshihiko Sakurai ${ }^{7}$ in 2014 found prolonged PT and APTT before IVIG were significantly shortened after IVIG, and elevated levels of FDP and D-dimer were significantly decreased. Similar findings were observed in studies from Michio Sakai et $\mathrm{al}^{10}$ in 2001 and Goshgar Mammadov et $\mathrm{al}^{26}$ in 2019. In addition, a previous study conducted by Toshihiko Imamura ${ }^{12}$ in 2005 showed that increased D-dimer concentrations are a useful clinical marker to distinguish atypical KD from bacterial cervical lymphadenitis. Moreover, several studies have proved that elevation of D-dimer could be a risk factor for CALs in KD. ${ }^{8,9}$ However, to our knowledge, only two studies have previously determined the roles of coagulation profiles in initial IVIG resistance prediction. Yoshihiko Sakurai et $\mathrm{al}^{7}$ in 2014 failed to find significant differences in coagulation profiles on patients responsive to initial IVIG $(n=13)$ and those refractory to it $(n=7)$ except for those with PT-INR. Thereafter, Wei-Xing Kong ${ }^{8}$ in 2019 found PT, APTT, TT, and fibrinogen were not significantly different between the IVIG-resistant group and IVIG-responsive group. Nonetheless, the D-dimer was remarkably elevated in the IVIG-resistant group. D-dimer level had a sensitivity of $87.0 \%$ and a specificity of $56.3 \%$ for predicting IVIG resistance at a cutoff point of $1.09 \mathrm{mg} / \mathrm{L}$. These observations are partly in line with our findings. However, both studies were limited by small sample size and retrospective design. Furthermore, the predictive value of ATIII for IVIG resistance has never been explored before. Given the sufficient number of patients and a prospective approach, the findings in our report might be more conclusive.

In terms of the repeated IVIG resistance prediction in KD, paucity of data was currently available and the role of coagulation profiles in this issue has never been investigated. Although several clinical trials $^{2,27}$ from Japan documented the addition of corticosteroid or ciclosporin therapy to standard-dose IVIG and aspirin in the primary therapy of KD reduced the initial non-response rate and decrease the incidence of CALs among high-risk patients for initial IVIG resistance predicted by Kobayashi, ${ }^{28}$ Sano, ${ }^{29}$ and Egami ${ }^{30}$ scores, the nonresponse rate still remained approximately $10 \%-20 \% .{ }^{31}$ These findings suggested that high-risk KD patients for IVIG resistance might mostly benefit from aggressive therapy and prediction of repeated IVIG resistance was equally essential and clinically significant. In the present study, we firstly found the repeated IVIG non-responders presented with a remarkably lower serum ATIII level compared with responders.
A cutoff value of $68.5 \%$ for repeated non-responders yielded a relatively moderate sensitivity of $71 \%$ and specificity of $55 \%$. Obviously, we could not identify all the non-responders for repeated IVIG by detecting serum ATIII level, and these data, however, may expand the limited information regarding repeated IVIG resistance prediction and provide some references for clinical management.

The strengths of this study were its prospective design and relatively large sample size. However, the present study has several limitations. First, this study was performed at a single institution. Our hospital is the largest pediatric medical center in Southwest China, which may lead to a selection bias due to a higher number of severely ill patients being admitted to this facility. Second, the present study was a prospective cohort study with strict inclusion and exclusion criteria. The findings of this study are, therefore, applicable only to Chinese patients with KD receiving standardized IVIG treatment $(2 \mathrm{~g} / \mathrm{kg})$ within 10 days of fever onset.

Despite these limitations, this prospective study is the first to report pronounced changes in TT, PT, APTT, FDP, D-dimer levels, INR, and ATIII activity in the acute stage of KD, which may serve as complementary laboratory biomarkers for predicting IVIG resistance. In addition, the predictive validity of ATIII activity as a single biomarker for IVIG resistance may be superior to other coagulation biomarkers with a relatively high sensitivity.

\section{5 | CONCLUSIONS}

In summary, the inflammatory process mediated by KD is associated with the cause of impaired coagulation, which supports the notion that patients with KD who also have hypercoagulation during the acute phase could be at higher risk of developing IVIG resistance.

\section{CONFLICT OF INTEREST}

The authors have no conflicts of interest to disclose.

\section{AUTHOR CONTRIBUTION}

Shuran Shao: Conceptualization (equal); Data curation (lead); Formal analysis (lead); Investigation (lead); Methodology (lead); Resources (equal); Writing-original draft (lead). Lixia Yang: Data curation (equal); Investigation (equal); Resources (equal); Supervision (lead); Validation (lead). Xiaoliang Liu: Data curation (equal); Investigation (equal); Resources (equal). Lei Liu: Data curation (equal); Resources (equal). Mei Wu: Data curation (equal); Resources (equal). Yuxin Deng: Resources (equal). Hongyu Duan: Resources (equal). Yifei Li: Funding acquisition (equal); Resources (equal). Yimin Hua: Conceptualization (equal); Funding acquisition (equal); Resources (equal). Lili Luo: Funding acquisition (equal). Kaiyu Zhou: Conceptualization (equal); Methodology (equal); Resources (equal); Supervision (lead); Validation (lead). Chuan Wang: Conceptualization (lead); Funding acquisition (lead); Project administration (lead); Supervision (equal); Validation (equal); Writing-review \& editing (lead). 


\section{DATA AVAILABILITY STATEMENT}

All data generated or analyzed during this study are included in this published article.

\section{ORCID}

Chuan Wang iD https://orcid.org/0000-0001-5165-8273

\section{REFERENCES}

1. Fukazawa R, Kobayashi J, Ayusawa M, et al. JCS/JSCS 2020 guideline on diagnosis and management of cardiovascular sequelae in kawasaki disease. Circulation J. 2020;84(8):1348-1407.

2. Kobayashi T, Kobayashi T, Morikawa A, et al. Efficacy of intravenous immunoglobulin combined with prednisolone following resistance to initial intravenous immunoglobulin treatment of acute Kawasaki disease. J Pediatr. 2013;163(2):521-526.

3. Son MB, Gauvreau K, Ma L, et al. Treatment of Kawasaki disease: analysis of 27 US pediatric hospitals from 2001 to 2006 . Pediatrics. 2009;124(1):1-8.

4. Levi M, van der Poll T, ten Cate H, van Deventer SJ. The cytokinemediated imbalance between coagulant and anticoagulant mechanisms in sepsis and endotoxaemia. Eur J Clin Invest. 1997;27(1):3-9.

5. Ogura T, Nakamura Y, Takahashi K, Nishida K, Kobashi D, Matsui S. Treatment of patients with sepsis in a closed intensive care unit is associated with improved survival: a nationwide observational study in Japan. J Intensive Care. 2018;6:57.

6. Dhar JP, Andersen J, Essenmacher L, Ager J, Bentley G, Sokol RJ. Thrombophilic patterns of coagulation factors in lupus. Lupus. 2009;18(5):400-406.

7. Sakurai Y, Takatsuka H, Onaka M, Takada M, Nishino M. Persistent endothelial damage after intravenous immunoglobulin therapy in Kawasaki disease. Inter Arch Allergy Immunol. 2014;165(2):111-118.

8. Kong WX, Ma FY, Fu SL, et al. Biomarkers of intravenous immunoglobulin resistance and coronary artery lesions in Kawasaki disease. World J Pediatr. 2019;15(2):168-175.

9. Masuzawa Y, Mori M, Hara T, Inaba A, Oba MS, Yokota S. Elevated $D$-dimer level is a risk factor for coronary artery lesions accompanying intravenous immunoglobulin-unresponsive Kawasaki disease. Therap Apheresis Dia. 2015;19(2):171-177.

10. Sakai M, Asayama K, Otabe T, Kohri T, Shirahata A. Low tissue plasminogen activator relative to plasminogen activator inhibitor-1 as a marker of cardiac complication in children with Kawasaki disease. Clin Appl Throm. 2001;7(3):214-218.

11. Zhou Y, Wang S, Zhao J, Fang P. Correlations of complication with coronary arterial lesion with VEGF, PLT, D-dimer and inflammatory factor in child patients with Kawasaki disease. Eur Rev Med Pharmacol Sci. 2018;22(16):5121-5126.

12. Imamura T, Yoshihara T, Yokoi K, Nakai N, Ishida H, Kasubuchi $Y$. Impact of increased D-dimer concentrations in Kawasaki disease. Eur J Pediatr. 2005;164(8):526-527.

13. Newburger JW, Takahashi M, Gerber MA, et al. Diagnosis, treatment, and long-term management of Kawasaki disease: a statement for health professionals from the Committee on Rheumatic Fever, Endocarditis, and Kawasaki Disease, Council on Cardiovascular Disease in the Young, American Heart Association. Pediatrics. 2004;114(6):1708-1733.

14. Sakurai Y. Autoimmune aspects of kawasaki disease. J Investig Allergol Clin Immunol. 2019;29(4):251-261.

15. Okada S, Hasegawa S, Suzuki Y, et al. Acute pericardial effusion representing the TNF- $\alpha$-mediated severe inflammation but not the coronary artery outcome of Kawasaki disease. Scand J Rheumatol. 2015;44(3):247-252.

16. Li Y, Zheng Q, Zou L, et al. Kawasaki disease shock syndrome: clinical characteristics and possible use of IL-6, IL-10 and IFN- $\gamma$ as biomarkers for early recognition. Pediatric Rheumatol. 2019;17(1):1.
17. Hu P, Jiang GM, Wu Y, et al. TNF- $\alpha$ is superior to conventional inflammatory mediators in forecasting IVIG nonresponse and coronary arteritis in Chinese children with Kawasaki disease. Clin Chim Acta. 2017;471:76-80.

18. Foley JH, Conway EM. Cross talk pathways between coagulation and inflammation. Circ Res. 2016;118(9):1392-1408.

19. Selleri C, Sato T, Anderson S, Young NS, Maciejewski JP. Interferongamma and tumor necrosis factor-alpha suppress both early and late stages of hematopoiesis and induce programmed cell death. J Cell Physiol. 1995;165(3):538-546.

20. Chelazzi C, Villa G, Mancinelli P, De Gaudio AR, Adembri C. Glycocalyx and sepsis-induced alterations in vascular permeability. Crit Care. 2015;19(1):26.

21. Reitsma S, Slaaf DW, Vink H, van Zandvoort MA, oude Egbrink MG. The endothelial glycocalyx: composition, functions, and visualization. Pflugers Arch. 2007;454(3):345-359.

22. Ohnishi Y, Yasudo H, Suzuki Y, et al. Circulating endothelial glycocalyx components as a predictive marker of coronary artery lesions in Kawasaki disease. Int J Cardiol. 2019;292:236-240.

23. Tripodi A, Mannucci PM. The coagulopathy of chronic liver disease. N Engl J Med. 2011;365(2):147-156.

24. Chen X, Zhao ZW, Li L, et al. Hypercoagulation and elevation of blood triglycerides are characteristics of Kawasaki disease. Lipids Health Dis. 2015;14:166.

25. Yoshizawa H, Nogami K, Matsumoto T, et al. Dynamic evaluation of hemostasis in the acute phase of Kawasaki disease using comprehensive coagulation functional assays. Thromb Res. 2019;174:76-83.

26. Mammadov G, Liu HH, Chen WX, et al. Hepatic dysfunction secondary to Kawasaki disease: characteristics, etiology and predictive role in coronary artery abnormalities. Clin Experi Med. 2020;20(1):21-30.

27. Hamada $\mathrm{H}$, Suzuki $\mathrm{H}$, Onouchi $\mathrm{Y}$, et al. Efficacy of primary treatment with immunoglobulin plus ciclosporin for prevention of coronary artery abnormalities in patients with Kawasaki disease predicted to be at increased risk of non-response to intravenous immunoglobulin (KAICA): a randomised controlled, open-label, blindedendpoints, phase 3 trial. Lancet. 2019;393(10176):1128-1137.

28. Kobayashi $\mathrm{T}$, Inoue $\mathrm{Y}$, Takeuchi K, et al. Prediction of intravenous immunoglobulin unresponsiveness in patients with Kawasaki disease. Circulation. 2006;113(22):2606-2612.

29. Sano T, Kurotobi S, Matsuzaki K, et al. Prediction of nonresponsiveness to standard high-dose gamma-globulin therapy in patients with acute Kawasaki disease before starting initial treatment. Eur J Pediatr. 2007;166(2):131-137.

30. Egami K, Muta H, Ishii M, et al. Prediction of resistance to intravenous immunoglobulin treatment in patients with Kawasaki disease. J Pediatr. 2006;149(2):237-240.

31. Durongpisitkul K, Soongswang J, Laohaprasitiporn D, Nana A, Prachuabmoh C, Kangkagate C. Immunoglobulin failure and retreatment in Kawasaki disease. Pediatr Cardiol. 2003;24(2):145-148.

\section{SUPPORTING INFORMATION}

Additional supporting information may be found online in the Supporting Information section.

How to cite this article: Shao S, Yang L, Liu X, et al. Predictive value of coagulation profiles for both initial and repeated immunoglobulin resistance in Kawasaki disease: $\mathrm{A}$ prospective cohort study. Pediatr Allergy Immunol. 2021;32:1349-1359. https://doi.org/10.1111/pai.13495 